\title{
Transformed lymphoplasmacytic lymphoma involving the main carina: A case report
}

\author{
MAKOTO NAKAO, TETSUYA OGURI, MIKINORI MIYAZAKI, HISATOSHI HIJIKATA, \\ MIDORI YOKOYAMA, EIJI KUNII, TAKEHIRO UEMURA, OSAMU TAKAKUWA, \\ HIROTSUGU OHKUBO, KEN MAENO and AKIO NIIMI
}

Department of Medical Oncology and Immunology, Nagoya City University, School of Medical Sciences, Nagoya, Aichi 467-8601, Japan

Received December 28, 2012; Accepted May 14, 2013

DOI: $10.3892 / 01.2013 .1369$

\begin{abstract}
A 41-year-old male was admitted to Nagoya City University Hospital subsequent to experiencing a cough with bloody sputum for a few days. The patient had a 4-year history of lymphoplasmacytic lymphoma (LPL) and had achieved a good partial response to anticancer chemotherapy. Chest computed tomography (CT) showed an endobronchial tumor of the main carina. A bronchoscopy revealed an exophytic tumor at the main carina, and autofluorescence imaging bronchovideoscopy showed that the tumor and surrounding area were magenta in color. The biopsy specimens demonstrated that the endobronchial tumor was composed of large atypical lymphoid cells. The patient was diagnosed with a high-grade transformation of LPL. In addition to describing a rare case of transformed LPL involving the main carina, the present study also summarizes and discusses endobronchial lymphomas, with a brief review of a number of published studies.
\end{abstract}

\section{Introduction}

Malignant lymphomas (ML) frequently involve intrathoracic organs, but endobronchial involvement (EBI) is relatively rare (1-3). The present study describes the case of a patient with lymphoplasmacytic lymphoma (LPL) transforming to large cell lymphoma, characterized by an endobronchial tumor of the main carina. To the best of our knowledge, this type of progression of LPL has not previously been reported. Notably, the present study used an autofluorescence imaging (AFI) bronchovideoscope system to evaluate the invasion of ML into the surrounding mucosa. The study provides further insight into the clinical significance of the EBI of ML and the

Correspondence to: Dr Tetsuya Oguri, Department of Medical Oncology and Immunology, Nagoya City University, School of Medical Sciences, 1 Kawasumi, Mizuho-cho Mizuho-ku, Nagoya, Aichi 467-8601, Japan

E-mail: t-oguri@med.nagoya-cu.ac.jp

Key words: endobronchial tumor, lymphoplasmacytic lymphoma, transformation, autofluorescence bronchoscopy utility of bronchoscopic examination in these patients. Written informed consent was obtained from the patient.

\section{Case report}

A 41-year-old male was referred to the Respiratory Division of Nagoya City University Hospital subsequent to experiencing a cough with bloody sputum for a few days. The patient had a 4-year history of a low-grade non-Hodgkin's lymphoma (NHL) with $\operatorname{IgM}$ paraproteinemia, diagnosed as LPL. The primary LPL lesion was in the bone marrow, with no lesions detected on computed tomography (CT) or 18F-fluorodeoxyglucosepositron emission tomography (FDG-PET). The patient had been treated with chemotherapy, including 8 courses of R-COP therapy (rituximab, cyclophosphamide, oncovin and prednisolone), 12 courses of fludarabine and 5 courses of bendamustine, and had attained a good partial response (PR). The cough with bloody sputum was first noticed $\sim 1$ month after the final dose of chemotherapy.

A physical examination revealed crackles in each lung and a oxyhemoglobin saturation of $96 \%$ in normal room air. A CT scan showed subcarinal lymphadenopathy and an endobronchial tumor involving the main carina (Fig. 1). The patient's platelet count was low $[63,000 / \mu 1$ (normal, $>148,000 / \mu 1)]$, and the concentration of circulating interleukin-2 receptor (IL-2R) was slightly elevated [538 U/ml (normal, <519 U/ml)]. The lactate dehydrogenase ( $\mathrm{LDH})$ level was within the normal range. The $\mathrm{IgG}, \mathrm{IgA}$ and $\mathrm{IgM}$ concentrations were $408 \mathrm{mg} / \mathrm{dl}$ (normal, 870-1700 mg/dl), $11 \mathrm{mg} / \mathrm{dl}$ (normal, 110-410 mg/dl) and $187 \mathrm{mg} / \mathrm{dl}$ (normal, 35-220 mg/dl), respectively. A bronchoscopic examination showed an exophytic tumor with rough surfaces and a white, moss-like appearance at the main carina (Fig. 2). An autofluorescence imaging (AFI) examination showed that the exophytic tumor and surrounding area were magenta in color. A transbronchial biopsy (TBB) specimen showed large atypical cells (Fig. 3). Immunohistochemical staining was positive for CD20 and CD79a and negative for CD3 and CD10. The cells were weakly positive for IgM and the $\kappa / \lambda$ ratio was high. The patient was diagnosed with a high-grade transformation of LPL involving the main carina.

The patient was treated with 2 courses of DeVIC therapy (dexamethasone, etoposide, ifosfamide and carboplatin), and 


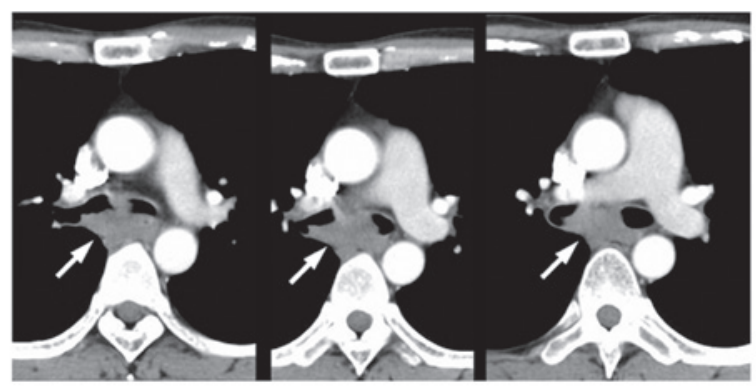

Figure 1. Contrast-enhanced computed tomography (CT) scan on admission showing subcarinal lymphadenopathy and an endobronchial tumor at the main carina.
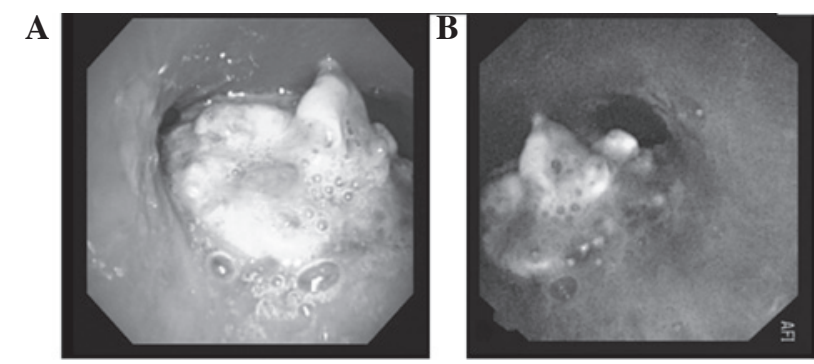

Figure 2. (A) Bronchoscopic photograph showing an exophytic tumor with a rough surface and a white moss-like appearance at the main carina. (B) Bronchovideoscope system autofluorescence imaging (AFI) revealed an exphytic tumor.
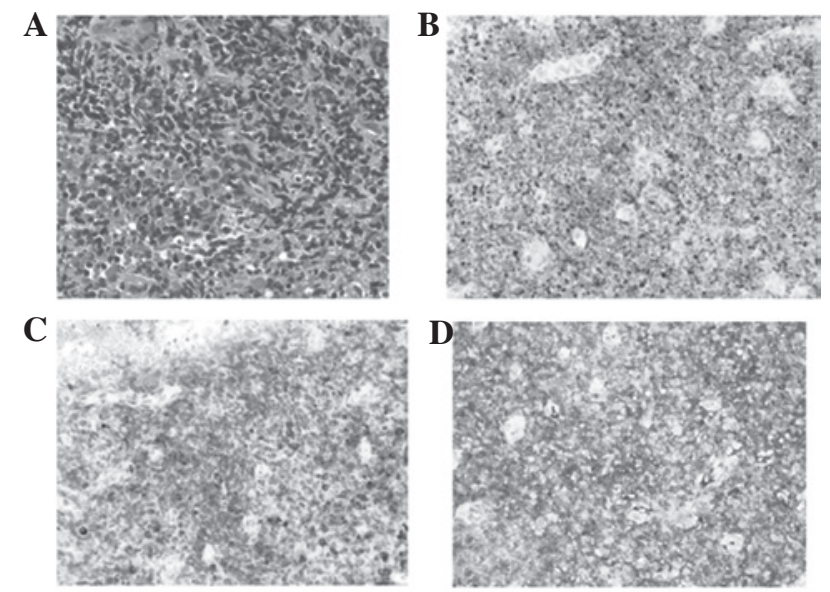

Figure 3. (A) Biopsy specimen from the endobronchial lesion (hematoxylin and eosin staining). (B) Immunohistochemical staining with anti-CD20 is positive. (C) Immunohistochemical staining with anti-CD79a is positive. (D) Immunohistochemical staining with anti- $\kappa$ is positive (Magnification, $\mathrm{x} 400$ ). In immunohistochemical staining, the darker areas represent positive staining.

achieved a complete response (CR), as confirmed by FDG-PET. Thereafter, the patient underwent an allogeneic stem cell transplantation and is currently (15 months post-treatment) alive without recurrence.

\section{Discussion}

The present study describes a rare case of transformed LPL with carinal involvement. Although ML frequently involves the intrathoracic organs, endobronchial involvement (EBI) in NHL is rare, even in patients with advanced disease $(3,4)$. To the best of our knowledge, a high-grade transformation of LPL detected as an endobronchial tumor has not previously been reported.

LPL is classified as a low-grade lymphoma and constitutes $<5 \%$ of all NHLs. LPL occurs in older adults, usually involving the bone marrow, lymph nodes and spleen, while extranodal involvement and a leukemic phase are rare (5). LPL tumors consist of a diffuse arrangement of small B lymphocytes with variable degrees of plasmacytoid differentiation. The clinical presentation usually consists of a disseminated disease, with $>20 \%$ of patients having monoclonal $\mathrm{IgM}$ paraproteinemia and hyperviscosity symptoms (5). The median survival of patients with LPL is 50-60 months due to the transformation to large cell lymphoma. The primary LPL lesion in the present patient was in the bone marrow, and IgM paraproteinemia was detected. The transformation to large cell lymphoma occurred $\sim 48$ months after the initial diagnosis of LPL. With the exception of the age of onset of the LPL and EBI, the patient had a typical clinical course.

Endobronchial lymphomas have been classified into 2 types; diffuse submucosal infiltrations in the presence of intra- and extra-thoracic lymphoma (type I), and central airway involvement due to a solitary mass in the absence of clinicallyapparent systemic lymphoma (type II) (6,7). In Japan, however, endobronchial lymphomas have been classified into 3 types, characterized by a raised tumor mass (type I), multiple submucosal nodules (type II) and diffuse submucosal infiltration (type III) (2). The present patient may be classified endoscopically as type II according to the former classification system and as type I according to the latter. Although the more common type of EBI accompanying NHL differs in various studies $(2,6,7)$, this may be due to the varying characteristics of NHL.

Five mechanisms of endobronchial metastasis have been proposed: i) direct bronchial invasion of a parenchymal mass; ii) direct bronchial invasion of a mediastinal mass; iii) lymphatic spread to peribronchial connective tissues; iv) transbronchial aspiration of tumor emboli; and v) direct hematogenous metastasis (8). The most common mechanisms regarding endobronchial NHLs are mechanisms ii) and iii) (8). Chest CT scans in the present patient showed that the endobronchial tumor was from mediastinal lymphadenopathy, suggestive of mechanism ii).

The majority of EBIs occur in patients with systemic or relapsed/refractory disease, and are more frequent in patients with Hodgkin's disease than in those with NHL $(2,7)$. Although the most frequent EBI loci observed in NHL patients differ, the main and lobar bronchi are the most common sites $(2,4,7)$. The most common histological subtypes of endobronchial lymphoma are considered to be bronchus-associated lymphoid tissue lymphoma and diffuse B-cell lymphoma $(6,7,9)$, although too few patients have been assessed to draw definitive conclusions. Further investigations of EBI accompanying NHL are warranted.

Autofluorescence bronchoscopy is an important tool in the early detection of preinvasive bronchial lesions, including squamous dysplasia, carcinoma in situ and early hilar lung carcinoma (10). A new type of autofluorescence bronchoscopy, AFI, has been revealed to more precisely distinguish preinvasive bronchial lesions and bronchitis (10). The AFI examination 
of the present patient demonstrated that the exophytic tumor and its surrounding areas were magenta in color. Since the mucosal surface surrounding the tumor was endoscopically smooth, submucosal invasion of the lymphoma was suspected. However, as only a few studies to date have described the use of AFI in patients with endobronchial lymphoma $(11,12)$, further studies are necessary to assess the usefulness of AFI in ML patients with EBI.

In conclusion, the present study describes a rare case of LPL involving the main carina. Bronchoscopic examinations with TBB and AFI led to a diagnosis of a transformation to large cell lymphoma. These findings may provide further insight into endobronchial lymphoma and, particularly in patients with refractory low-grade lymphoma, emphasize the importance of histological examination of newly developed lesions.

\section{References}

1. Banks DE, Castellan RM and Hendrick DJ. Lymphocytic lymphoma recurring in multiple endobronchial sites. Thorax 35 : 796-797, 1980

2. Tanigawa S, Hosokawa Y, Abe M, et al: Classification and clinical features of endobronchial involvement of non-Hodgkin's lymphoma. JJSRE 14: 15-21, 1992 (In Japanese).

3. Fujimoto Y, Nomura K, Shimizu D, et al: Pulmonary relapse of non-Hodgkin's lymphoma in bilateral upper lobes. Intern Med 45: 971-973, 2006.
4. Eng J and Sabanathan S: Endobronchial non-Hodgkin's lymphoma. J Cardiovasc Surg (Torino) 34: 351-354, 1993.

5. Vitolo U, Ferreri AJ and Montoto S: Lymphoplasmacytic lymphoma-Waldenstrom's macroglobulinemia. Crit Rev Oncol Hematol 67: 172-185, 2008.

6. Rose RM, Grigas D, Strattemeir E, Harris NL, Linggood RM. Endobronchial involvement with non-Hodgkin's lymphoma. A clinical-radiologic analysis. Cancer 57: 1750-1755, 1986.

7. Solomonov A, Zuckerman T, Goralnik L, Ben-Arieh Y, Rowe JM and Yigla M: Non-Hodgkin's lymphoma presenting as an endobronchial tumor: report of eight cases and literature review. Am J Hematol 83: 416-419, 2008.

8. Kilgore TL and Chasen MH: Endobronchial non-Hodgkin's lymphoma. Chest 84: 58-61, 1983.

9. Gómez-Román JJ, Pérez-Montes R, Pérez-Expósito MA, Richard C, Baro J and Val-Bernal JF: Primary lymphoplasmacytoid lymphoma of the trachea with immunoglobulin $G$ paraprotein. Pathol Int 49: 1100-1104, 1999.

10. Chiyo M, Shibuya K, Hoshino H, et al: Effective detection of bronchial preinvasive lesions by a new autofluorescence imaging bronchovideoscope system. Lung Cancer 48: 307-313, 2005.

11. Sato C, Suzuki H, Watanabe M, Kojima K, Tsuchida F and Takeda H: Spontaneous regression of mucosa-associated lymphoid tissue lymphoma of the lung. Nihon Kokyuki Gakkai Zasshi 48: 677-682, 2010 (In Japanese).

12. Ueda Y, Matsuo K, Tsushima M, Fujiwara K, Yonei T and Sato T: A case showing tracheal origin of MALT (mucosa-associated lymphoid tissue) lymphoma that could be observed with autofluoresence imaging bronchovideoscope before and after treatment JJSRE 32: 162-168, 2010 (In Japanese). 